\title{
Zur Altersstellung der Torfe und Gyttjen von Herxheim, Jockgrim und Rheinzabern in der Vorderpfalz
}

\author{
Von Ilse Peters, Bonn \\ Mit 6 Abbildungen und 1 Tabelle im Text
}

$\mathrm{Zusammenfassung.} \mathrm{Die} \mathrm{in} \mathrm{den} \mathrm{Tongruben} \mathrm{Herxheim,} \mathrm{Jockgrim} \mathrm{und} \mathrm{Rheinzabern}$ vorkommenden Torfe und Gyttjen wurden pollenanalytisch untersucht, um dem immer noch nicht geklärten Datierungsproblem der dort angeschnittenen Pleistozänserien näher zu kommen.

Für die Ablagerung der Herxheimer Torfe ergab sich eine subarktische Klimaphase, die den Beginn oder das Ende einer Warmzeit, aber auch ein Interstadial während einer Kaltzeit bezeichnen kann. Die nähere Zuordnung zu einem bestimmten Interglazial bzw. Glazial ist nach den pollenanalytischen Ergebnissen allein jedoch nicht möglich.

Die Torfe und Gyttjen der nur wenige Kilometer voneinander entfernt liegenden Tongruben Jockgrim und Rheinzabern sind gleichaltrig. Die spurenweise in ihnen enthaltenen tertiären Gattungen Tsuga, Castanea, Pterocarya und Carya sowie auch Fagus sprechen für ein frühpleistozänes Alter der Torfe. $\mathrm{Da}$ aus dem süddeutschen Raum bisher noch keine vollständigen Pollendiagramme aus dem Altpleistozän vorliegen, ist die sichere stratigraphische Einordnung der Jockgrimer und Rheinzaberner Torfe vorläufig nicht möglich.

$\mathrm{Sum} \mathrm{m}$ a r y. In order to clarify the Pleistocene successions present in the clay-pits at Herxheim, Jockgrim and Rheinzabern in Vorderpfalz, a pollen analysis has been made of peat and silt deposits. The following results have been obtained:

1) The Herxheim peat-layers can be assigned to a subarctic phase, which may belong either at the beginning or end of any Interglacial or Interstadial. The pollen diagrams do not permit a definite dating.

2) The clay-pits of Jockgrim and Rheinzabern are located not far apart and the peat- and siltlayers are of the same age. They contain pollen of the Tertiary forms: Tsuga, Castanea, Pterocarya and Carya and also some Fagus. These indicate a lower Pleistocene age for the deposits. Up to now, no complete pollen diagrams are available for the southern German area. Therefore it is not possible at present to date definitely the deposits of Jockgrim and Rheinzabern.

\section{Einleitung}

Die in den Tongruben Herxheim, Jockgrim und Rheinzabern (Vorderpfalz) aufgeschlossenen Pleistozänserien sind bereits mehrfach Gegenstand stratigraphischer und paläontologischer Untersuchungen gewesen (SChwegler 1935, Plewe 1938, Kuss 1961). Dabei ist es jedoch bisher nicht gelungen, einen sicher datierbaren Leithorizont zu finden, der einen Hinweis für die Einstufung der übrigen Schichten geben würde.

Im Zuge einer vom Geographischen Institut Bonn durch Frau S. HAFFnER ausgeführten Dissertation, die sich mit der Quartärmorphologie der Vorderpfalz befaßt, übertrug mir Herr Professor Dr. Dr. h. c. C. Troll die pollenanalytische Bearbeitung der Torfe von Herxheim, Jockgrim und Rheinzabern.

Im Frühjahr 1963 wurde mit der Probenentnahme begonnen. Erst nachdem bereits ein Teil des gesammelten Materials bearbeitet war, erfuhr ich von den gleichlaufenden Untersuchungen von Herrn Dr. E. Kolumbe, Hamburg. Nach Rücksprache mit Dr. Kolumbe stellte ich meine Untersuchungen sofort ein. Da jedoch die Kenntnis der pollenanalytischen Ergebnisse für die Dissertation von S. HAFFNER von großem Interesse war, wurde auf Wunsch von Herrn Professor TRoLl die begonnene Arbeit wieder aufgenommen. Ich führte sie soweit fort, wie es für die Klärung des mir gestellten Fragenkomplexes notwendig war. Die hier mitgeteilten Ergebnisse sind somit nur als vorläufige zu werten. Die eingehende Bearbeitung der Torfe und Gyttjen erfolgt weiterhin durch Dr. Kolumbe, der über ein sehr reichhaltiges Material verfügt. Ich danke Herrn Dr. Kolumbe für seine gleich zu Anfang geäußerte, selbstlose Bereitwilligkeit, mich an der Bearbeitung der genannten Torfvorkommen teilnehmen zu lassen. Die Veröffentlichung der hier mitgeteilten Ergebnisse erfolgt mit seinem Einverständnis. 


\title{
I. Herxheim (Grube Speth)
}

Das in Abb. 1 in etwa 8 m Tiefe eingezeichnete Torfband ${ }^{1}$ ) war im Frühjahr 1963 in der Ostwand der Grube mit rd. $70 \mathrm{~cm}$ Mächtigkeit aufgeschlossen. Die Proben wurden, von der Oberkante des Torfes ausgehend, in Abständen von je $5 \mathrm{~cm}$ entnommen, außerdem eine Sammelprobe aus dem liegenden Ton, um die Pollenführung dieses Sedimentes zu prüfen.

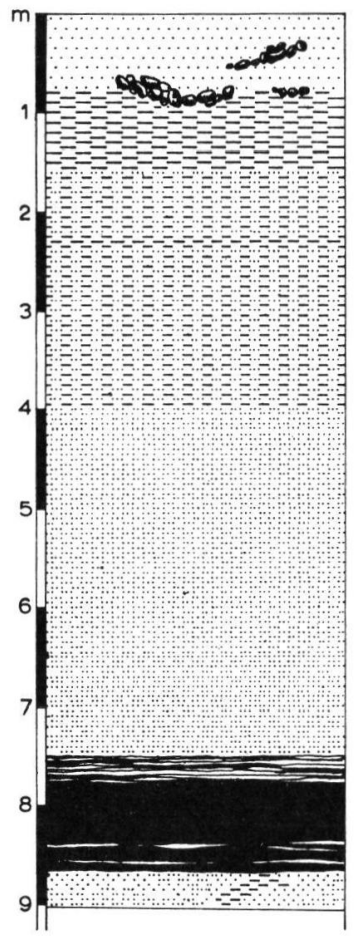

\author{
heller toniger Sand mit vereinzelten \\ Schottertaschen \\ fetter dunkelblaugrauer Ton \\ fettes grauviolettes Tonband \\ grauer schluffiger Ton mit Lößkomponente
}

verschwemmtes Lößmaterial

mit deutlicher Bänderung

Torfband

buntes, sandig bis toniges Material

Entwurf und Zeichnung: S. HAFFNER

Abb. 1. Profil der Ziegelei in Herxheim b. Landau. Entwurf und Zeichnung S. Hafrner.

Das Torfflöz, das als einzige Schicht von Herxheim pollenanalytisch untersucht wurde, enthielt über seine gesamte Höhe in wechselndem Maße tonig-schluffige Beimengungen, in den untersten $15 \mathrm{~cm}$ außerdem einen geringen Gehalt an Feinsand. Daher mußten fast alle Proben mit Flußsäure aufbereitet werden. Nur zwischen $37 \mathrm{~cm}$ und $47 \mathrm{~cm}$ unter seiner Oberfläche wurde der Torf so rein, daß sogar der Kalilauge-Aufschluß genügte.

In seinem unteren Drittel ließ sich der stark gepreßte Torf in Form einzelner, großer Platten aus dem Verband lösen. Nach oben hin wurde er im lufttrockenen Zustand je nach Tongehalt mehr oder weniger bröckelig. An keiner Stelle wurden größere Pflanzenreste vermerkt. Nach der mikroskopischen Durchmusterung handelt es sich um einen mit Gräsern und Cyperaceen durchsetzten Hypnum-Torf. Der Erhaltungszustand der Pollen wechselte; meist war er sehr schlecht. Zerrissene Pinus- und Picea-Pollen, die bei der Zählung aus ihren Bruchstücken zusammengesetzt werden mußten, waren keine Seltenheit. Das deutet auf eine beträchtliche mechanische Beanspruchung des Pollen-Niederschlags während oder nach seiner Ablagerung hin, wie es bereits der Schluffanteil des Torfes vermuten läßt. OOrtlich mag es auch zu Austrocknungen gekommen sein; denn selbst die sehr

1) Die stratigraphischen Skizzen wurden von S. HAFFNER angefertigt. 


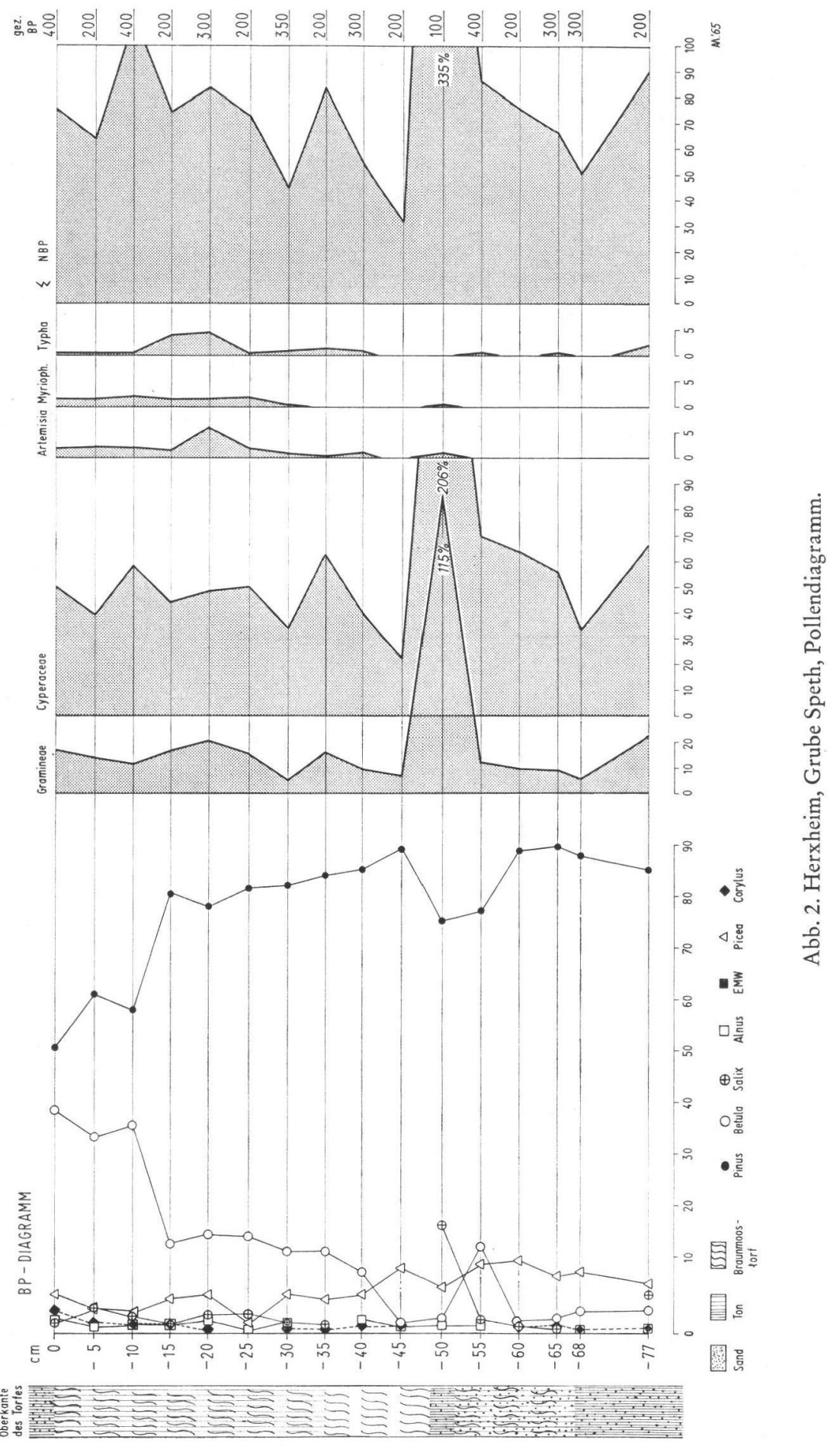


widerstandsfähigen Pinus-Pollen waren teilweise bis zur hyalinen Durchsichtigkeit zersetzt.

Offenbar veranschaulichen uns die Herxheimer Torfe mit ihren liegenden und hangenden Tonen einen zur Versumpfung fortschreitenden Fluß-Totarm, der immer wieder überflutet wird, bis schließlich das Wasser endgültig die Oberhand behält.

Obwohl der Probenabstand mit $5 \mathrm{~cm}$ für den stark gepreßten Herxheimer Torf ziemlich groß erscheint, wirkt er sich auf die Auswertung des Diagramms nicht störend aus, da die Kurven der einzelnen Pollenkomponenten sehr eintönig verlaufen (Abb. 2). Pinus herrscht im gesamten Diagramm weitaus vor. Ihre hohen, um 80\% schwankenden Werte werden erst in den drei obersten Spektren durch einen entsprechenden Vorstoß von Betula auf $60 \%$ und schließlich 50\% zurückgedrängt. Der sich sprunghaft vollziehende Umschwung deutet sich in den darunterliegenden Spektren bereits an.

$\mathrm{Da}$ im gesamten Profil nur ein einziges Mal $(-68 \mathrm{~cm})$ eine schlecht erhaltene Koniferen-Spaltöffnung gefunden wurde, muß der Pinus-Pollen auf Fernflug zurückgeführt werden. Gleiches gilt auch für alle übrigen Vertreter der Baumflora, vielleicht mit Ausnahme von Salix. Selbst Betula scheint kein unmittelbarer Bestandteil des versumpften Altwasserbereichs gewesen zu sein, denn die Suche nach Früchten und Kutikeln in den oberen Proben, die besonders reichlich ihre Pollen enthielten, blieb erfolglos.

Neben Pinus und Betula weist nur noch Picea eine geschlossene Kurve auf. Mit Werten bis knapp $10 \%$ ist sie die dritthäufigste Art in Herxheim. Die übrigen BP-Komponenten ${ }^{2}$ ) - Alnus, Corylus und Salix - bewegen sich in unterbrochenen Kurven ganz am linken Rand des Diagramms. Der Vorstoß von Salix in $-50 \mathrm{~cm}$ ist durch das Platzen einer zufällig mit der Probe aufgesammelten Anthere bedingt.

Die Ablagerung des Herxheimer Torfes hat sich nach diesen Befunden offenbar während einer recht kühlen, als subarktisch zu bezeichnenden Klimaphase vollzogen. Das äußert sich auch in der Krautflora (Tab.1): sämtliche wärmebedürftigen Typen fehlen auch hier, dafür treten Ephedra-Pollen (fragilis-Typ) auf.

Der subarktische Charakter der Herxheimer Flora bereitet Schwierigkeiten für ihre zeitliche Einordnung. Sie kann ebenso den Beginn wie den Endabschnitt irgendeines Interglazials bezeichnen oder auch ein Interstadial. Da der hangende Tonkomplex des Torfes gern der Günz-Kaltzeit zugewiesen wird, wäre die Deutung als günz-interstadial nicht abwegig. Die Entscheidung kann vielleicht durch die pollenanalytische Untersuchung der hangenden Tonschichten erbracht werden, eine zwar mühsame, aber doch lohnende Arbeit.

\section{Jockgrim und Rheinzabern}

Auch an diesen beiden, nur wenige Kilometer voneinander entfernten Orten liegen die Torfe in Tonschichten eingebettet tief unter der heutigen Oberfläche (Abb. 3 u. 5). In Jockgrim war im Frühjahr 1963 das durchlaufende, $50 \mathrm{~cm}$ mächtige Torfband in der Hauptabbauwand (SW-Wand) aufgeschlossen. Es enthielt im unteren Drittel eine etwa $10 \mathrm{~cm}$ mächtige Toneinlagerung, die sich als sehr pollenarm erwies und daher nicht statistisch ausgewertet wurde.

In Rheinzabern fehlte das obere, von Kolumbe 1960 beschriebene Torfband ganz. Nach einer Mitteilung von Frau HAFFnen war es bereits im Vorjahr nicht mehr durchgehend aufgeschlossen, sondern brach plötzlich ab (Abb. 3).

Von dem unteren Torfband war dicht unter seiner Oberkante eine schmale Humuszone durch eine $10-30 \mathrm{~cm}$ mächtige Tongyttja-Einlagerung abgespalten.

2) Es werden folgende Abkürzungen benutzt: $\mathrm{BP}=$ Baumpollen, $\mathrm{NBP}=$ Nichtbaumpollen, $\mathrm{EMW}=$ Eichenmischwald (Quercus + Ulmus + Tilia + Fraxinus). 
Ta be 11 e 1

Herxheim: Zusammenstellung der im Diagramm nicht aufgeführten Pollen und Sporen (in \% der BP).

\begin{tabular}{|c|c|c|c|c|c|c|c|c|c|c|}
\hline 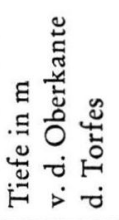 & 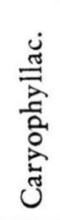 & 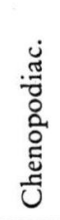 & 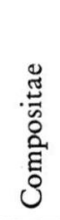 & 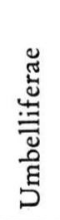 & 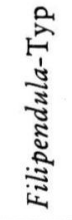 & 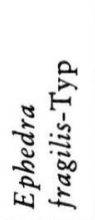 & 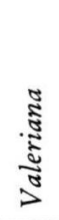 & 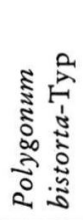 & 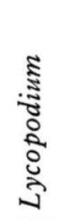 & 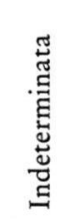 \\
\hline 0 & & & 0,5 & 0,5 & & & & & & 4,0 \\
\hline 0,05 & & & & & 1,5 & & & & & 5,5 \\
\hline 0,10 & & & & 0,5 & 24,0 & & 0,5 & & & 12,5 \\
\hline 0,15 & 1,0 & & 2,0 & & 0,5 & & & & & 3,5 \\
\hline 0,20 & & & 0,5 & & & 0,5 & & & 1,0 & 2,5 \\
\hline 0,25 & & & & & & & & 0,5 & & 2,5 \\
\hline 0,30 & & & 0,5 & 0,5 & & & & 0,5 & & 2,0 \\
\hline 0,35 & & & & & & & & & & 3,5 \\
\hline 0,40 & & 0,5 & & & 0,5 & & & & & 3,0 \\
\hline 0,45 & & & 0,5 & 1,0 & & & & & & 1,0 \\
\hline 0,50 & 2,0 & 1,0 & 5,0 & & & & & & 2,0 & 11,0 \\
\hline 0,55 & & & 0,5 & 0,5 & & & 0,5 & 0,5 & & 2,0 \\
\hline 0,60 & & & & & & & & & & 3,0 \\
\hline 0,65 & & & & & & 0,5 & 0,5 & & & 0,5 \\
\hline 0,67 & & & 0,5 & & & 0,5 & & & & \\
\hline 0,77 & & 0,5 & 2,5 & & & & & & & 1,0 \\
\hline
\end{tabular}




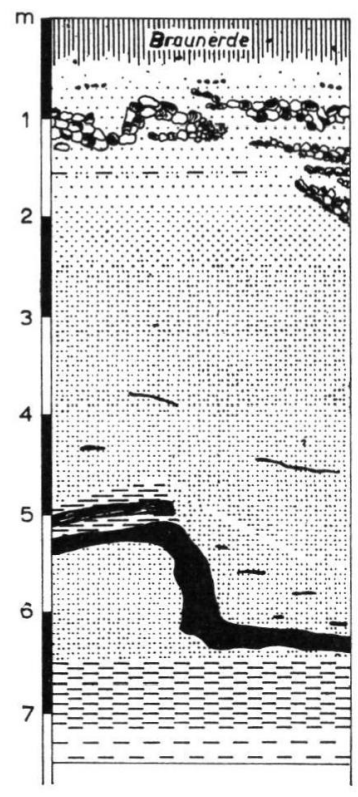

Flugsand

Rhein-Hardtfluß-Schotter

grober Sand mit zwischengeschalteter

Lehmlage

mittelfeiner Sand, deutlich geschichtet

verschwemmtes Lößmaterial

mit Bändern und Linsen pflanzlicher Reste

oberes, toniges Torfband, eingelagert

in fetten grauen Ton

verschwemmtes Lößmaterial,

z. T. mit pflanzlichen Resten

unteres Torfband

mausgrauer fetter Ton

hellgraublauer Ton

Entwurf und Zeichnung: S. HAFFnER

Abb. 3. Profil der Ziegeleigrube in Rheinzabern. Entwurf und Zeichnung S. Haffner.

Infolge der sehr schlechten Aufschlußverhältnisse wurden von Rheinzabern nur wenige Stichproben aus der unteren Torfzone entnommen. Von dem oberen Torfband und den es unter- bzw. überlagernden Tongyttjen erhielt ich je eine Probe aus den Aufsammlungen von Frau HAFFNER.

Die bereits stark zersetzten Torfe von Jockgrim und Rheinzabern ließen sich nur schwer charakterisieren. Sie enthielten verschiedenartige Blattfragmente sowie Holz- und Rindenreste, das untere Torfband von Rheinzabern auch Schilfrhizome, so daß die Bezeichnung "Schwemmtorf“ zutreffend erscheint. Bei der oberen humosen Zone handelt es sich nach der mir übergebenen Probe um eine Gyttja.

Die Pollen zeigten auch hier fortgeschrittene Korrossionserscheinungen. Es ist daher möglich, daß gewisse Formen mit wenig widerstandsfähiger Exine durch ihre schlechte Erhaltungsfähigkeit unzureichend oder vielleicht auch gar nicht erfaßt worden sind. Auch die Bestimmung war aus dem gleichen Grunde manchmal schwierig. So könnte es sich bei den als cf. Thalictrum bezeichneten Formen auch um Alisma handeln. Obwohl sich Alisma der Vergesellschaftung von Typha und Stratiotes besser einfügen würde, wurde Thalictrum der Vorzug gegben, da die morphologischen Merkmale doch insgesamt mehr für diese Gattung sprachen. Infolge ihres hohen Tongehaltes mußten auch die Jockgrimer und Rheinzaberner Proben mit Flußsäure aufbereitet werden; sie wurden anschließend azetolysiert.

Bei der Betrachtung des Jockgrimer Pollendiagramms (Abb.4) fällt ähnlich wie in Herxheim die führende Rolle von Pinus auf. Doch hat hier die BP-Flora einen ganz anderen Charakter. Abgesehen davon, daß die Werte von Pinus niedriger und die von Picea höher liegen als in Herxheim, tritt als weiterer Nadelbaum in dem obersten und in den beiden untersten Horizonten Abies mit über 20\% hinzu. Außerdem ist der EMW mit seinen Begleitern vorhanden - wenn auch nur in geringen Prozenten - und schließlich kommen spurenweise die tertiären Elemente Tsuga, Carya, Pterocarya und Castanea vor sowie ein Pollenkorn von Fagus. 


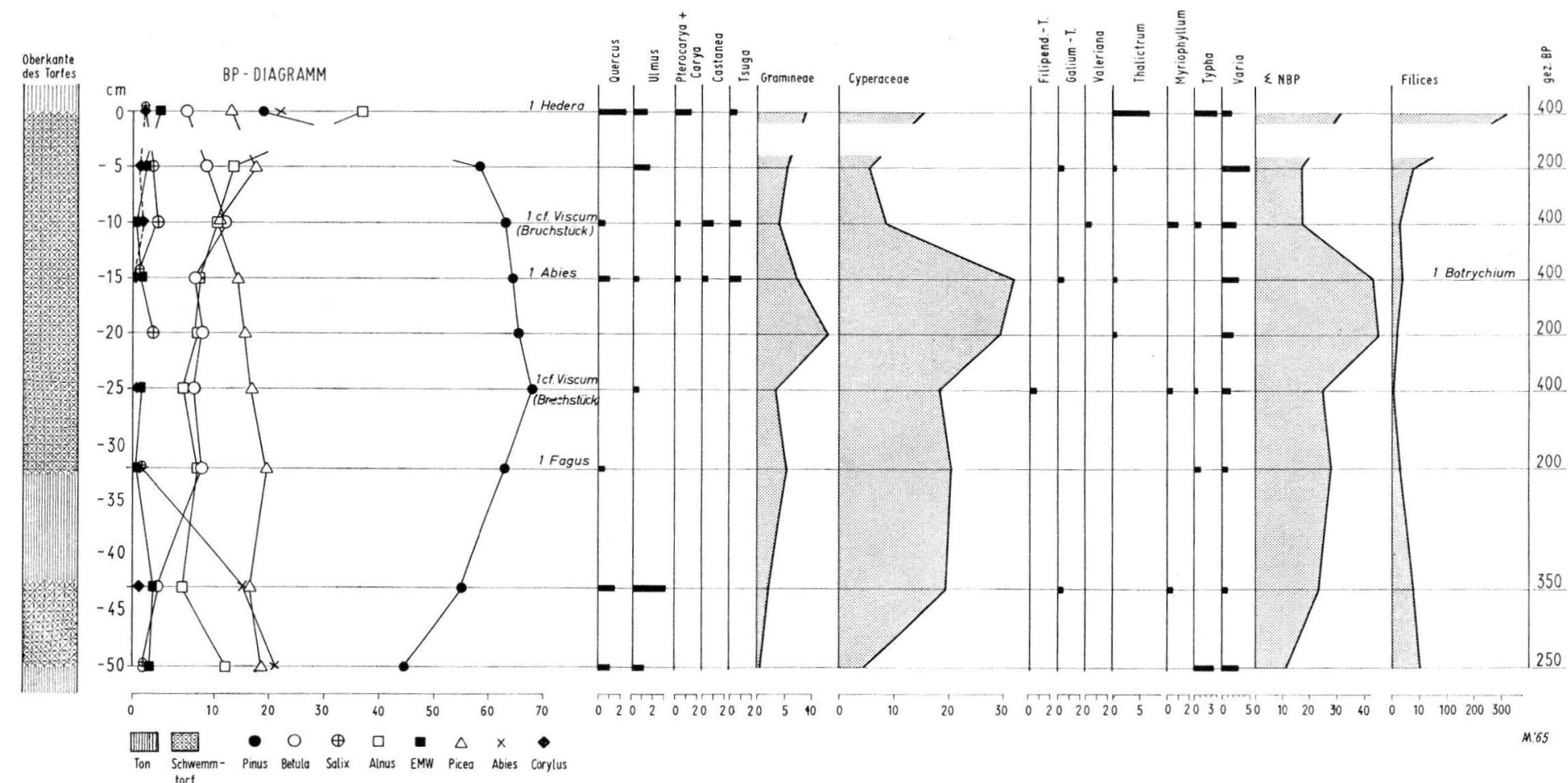

Abb. 4. Jockgrim, Pollendiagramm. 


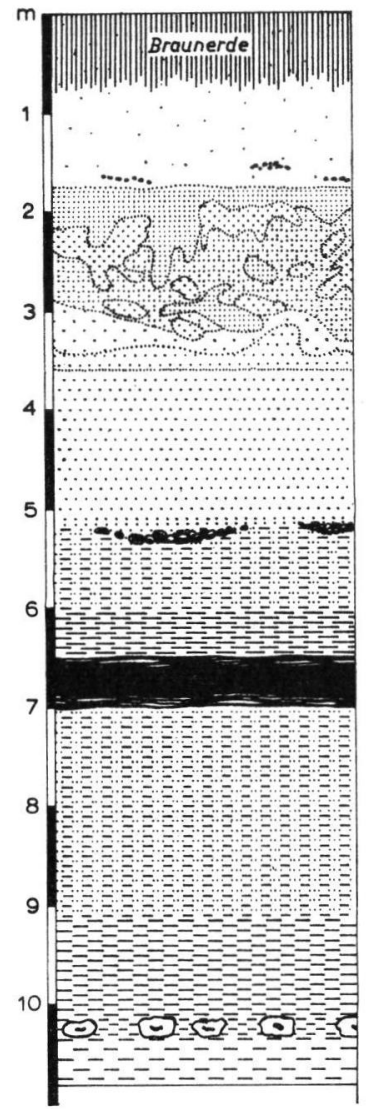

Flugsand

Rhein-Hardtfluß-Material;

Schichtlagerung durch Kryoturbations-

vorgänge aufgelöst

graugelber mittelfeiner Sand

Buntsandsteinschotter in $\mathrm{v} \in \mathrm{reinzelten}$ Taschen

hellblauer Ton mit Lößkomponente

fetter blauschwarzer Ton

Torfband

hellblauer Ton mit Lößkomponente

dunkelblaugrauer Ton

gelbliche Kalkanreicherungen

mit festem Kern

graublauer Ton

Entwurf und Zeichnung: S. HafFNeR

Abb. 5. Profil der Ziegeleigrube in Jockgrim. Entwurf und Zeichnung S. Haffner.

Zwischen den beiden obersten Horizonten liegt vermutlich ein Hiatus. Denn es steigen nicht nur die Abies-Werte sprunghaft von $0 \%$ auf $22 \%$ an, sondern auch Alnus erfährt einen beträchtlichen Vorstoß, während Pinus von 56\% auf $19 \%$ zurückgeht. Die Farnsporen steigen sogar von $8 \%$ auf $330 \%$ an!

In der Beschaffenheit des Sedimentes ließ sich makroskopisch kein Unterschied feststellen. Doch erwies sich die obere Probe im Gegensatz zur unteren als überaus pollenreich, wobei der Erhaltungszustand der Pollen ausnahmsweise gut war. Leider wurden keine Zwischenproben aus dem fraglichen Bereich entnommen, die diese Frage hätten klären können.

Ein steiler, aber offenbar synchroner Abies-Abfall liegt im unteren Teil des Profils vor. Der Rückgang der Tanne erfolgte hier wahrscheinlich während der zwischengeschalteten Tonsedimentation. -

Betrachtet man von den Rheinzaberner Pollenspektren (Abb.6) zunächst die der unteren Torfzone, so zeigt sich, daß das obere, abgespaltene Humusbändchen (Probe I) zeitlich mit dem eigentlichen Torfband zu vereinigen ist. Weiterhin lassen sich die Spektren von Probe II und III sehr genau mit den beiden obersten Horizonten von Jockgrim parallelisieren. Denn in Rheinzabern sind in gleicher Weise die sprunghaften Anderungen der Abies-, Alnus- und Pinus-Werte ausgebildet wie dort. Auch die Werte der übrigen BP sowie der Krautflora und - unter Einbeziehung von Probe I - auch der Filices lassen sich gut miteinander vergleichen. Vor allem finden wir in Rheinzabern die tertiären 


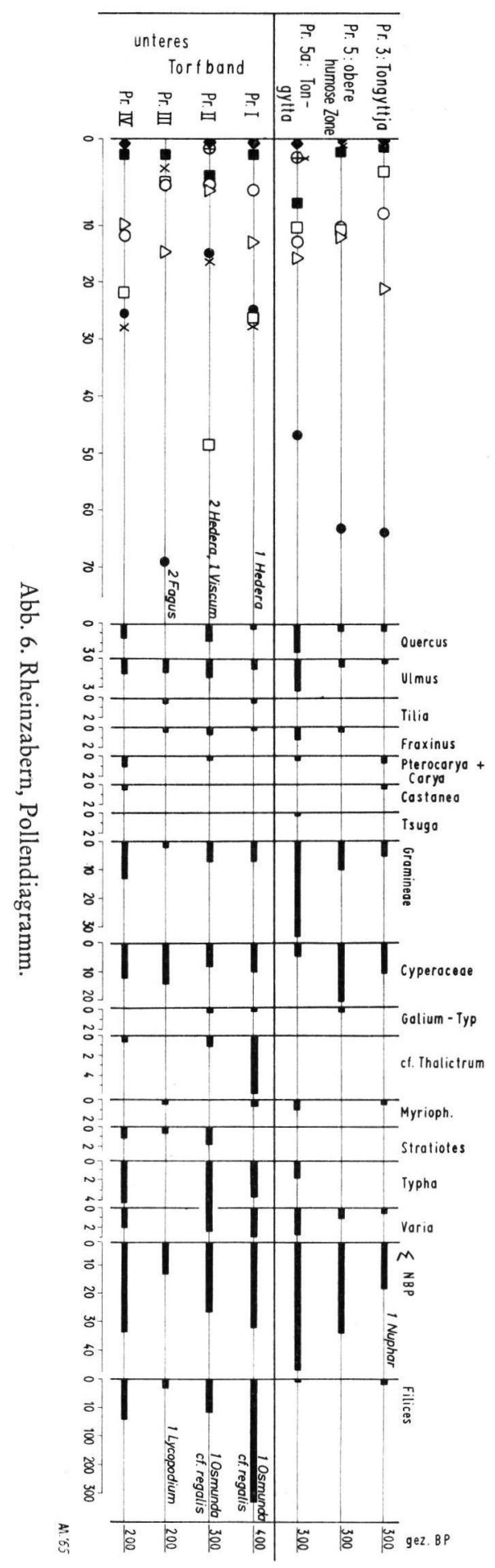


Formen und Fagus wieder, die in Jockgrim ebenfalls in den entsprechenden Horizonten vorkommen. Probe II war noch pollenreicher als die betreffende von Jockgrim; sie enthielt nur wenig organische Verunreinigungen und mußte zum Zählen sogar verdünnt werden; man kann das Sediment als interglazialen Fimmenit bezeichnen. Die Pollenerhaltung war auch hier außergewöhnlich gut.

Es dürfte wohl kein Zweifel darüber bestehen, daß die Torfe von Jockgrim und Rheinzabern gleichaltrig sind. Abweichend verhält sich nur Probe IV von Rheinzabern. $\mathrm{Da}$ sie direkt an der unteren Grenze vom Torf zum liegenden Ton entnommen wurde und das gesamte Torfband einen wahrscheinlich tektonisch bedingten, welligen Verlauf aufwies, darf man hier wohl eine Störung der Schichten als Erklärung annehmen.

Die obere Humuszone (Gyttja) läßt ebenso wie die sie dicht über- und unterlagernden Tone keine wesentliche Änderung in der Zusammensetzung der Pollenflora erkennen.

Für die zeitliche Einordnung der Jockgrimer und Rheinzaberner Torfe geben uns die auch im Altpleistozän noch vorkommenden, tertiären Gattungen Tsuga, Castanea, Pterocarya und Carya sowie die im Laufe des Altpleistozän zunächst aussterbende Buche (Fagus) einen Hinweis. Nach den sehr vollständigen und ausführlichen Diagrammen aus den Niederlanden (ZAGwIJN 1956-1963), die uns in einmaliger Weise den Florenwechsel vom ausgehenden Tertiär bis ins mittlere Pleistozän wiedergeben, wären Jockgrim und Rheinzabern dem frühen Pleistozän - wahrscheinlich Tegelen - zuzuordnen. Denn nach Z AGWiJN erreicht nur Tsuga manchmal noch den Beginn der Cromer-Warmzeit. Carya und Pterocarya sterben mit dem Waal-Interglazial aus, während Castanea und Fagus auf das Tegelen beschränkt bleiben, letztere sogar auf dessen tiefere Horizonte.

Zwischen den Niederlanden und der Vorderpfalz liegt jedoch eine Entfernung von mehreren hundert Kilometern, und es fragt sich, ob Parallelisierungen über so weite Strecken möglich sind. Versucht man, die in den Jockgrimer und Rheinzaberner Torfen überlieferten Vegetationsabschnitte in den holländischen Diagrammen wiederzufinden, so gelingt dies nicht. Zwar ist es immer schwierig, kurze Profilabschnitte in Vergleichsdiagramme einzuordnen, zumal wenn sie nicht ganz typisch sind; doch liegt hier noch ein anderer Grund vor: die hohen Abies-Werte von Jockgrim und Rheinzabern sind aus dem gesamten Altpleistozän der Niederlande wie auch aus den benachbarten deutschen Vorkommen unbekannt. Sie fehlen auch in der als tegelen-zeitlich angesehenen „kolchischen Phase" von Buchenau in Hessen (Leschik 1952) und finden sich erst in der den pfälzischen Vorkommen am nächsten gelegenen Schwanheimer Flora bei Frankfurt/M. wieder (BAAS 1932). Nach allgemeiner Ansicht wurde sie ebenfalls während der Tegelen-Warmzeit gebildet. Hier erinnern die beiden obersten Spektren des Pollendiagramms an die Verhältnisse von Jockgrim und Rheinzabern, weil die sonst bei etwa 5\% liegenden Abies-Werte plötzlich auf $27 \%$ und sogar $36 \%$ ansteigen. Diese hohen Werte sind gewiß nicht nur die Folge einer selektiven Zersetzung, wie sie BAAS aus dem Sedimentwechsel vom „Braunkohlenton" zum sandigen Ton erklärt. Gegen eine solche Annahme spricht der Verlauf der übrigen BP-Komponenten. ${ }^{3}$ ) Wahrscheinlich verbindet sich mit dem Sedimentationswechsel ein Hiatus - ähnlich wie in Jockgrim. Leider bricht hier das Diagramm ab, und der untere, hauptsächlich von Tsuga beherrschte Teil läßt keine weiteren Vergleiche zu.

So spärlich die bisherigen Befunde auch sind, deuten sie doch darauf hin, daß innerhalb Mitteleuropas während des Altpleistozäns ein differenziertes Florenbild bestand. Auch wird man mit Verschiebungen im zeitlichen Aussterben der tertiären Gattungen zwischen Nord und Süd rechnen müssen. Daher bleibt das genaue Alter der Torfe von Jockgrim und Rheinzabern solange problematisch, wie noch keine weiteren, vollständigeren Vergleichsdiagramme aus dem süddeutschen Raum vorliegen, die entweder mit Hilfe geologischer Methoden sicher datierbar sind oder sich mit den niederländischen

3) Der Abfall der Abies-Werte in den beiden untersten Spektren läßt sich jedoch zwanglos durch selektive Zersetzung erklären. 
Diagrammen einwandfrei konnektieren lassen. Wir dürfen wohl mit großer Wahrscheinlichkeit annehmen, daß die Torfe von Jockgrim und Rheinzabern älter als Cromer sind und auf die Übereinstimmung der hohen Abies-Werte mit denen der beiden obersten Horizonte von Schwanheim hinweisen. Nähere Aussagen sind unserem heutigen Wissensstand gemäß jedoch nicht vertretbar.

\section{Lit e r a t u :}

BAAs, J.: Eine frühdiluviale Flora im Mainzer Becken. - Ztschr. f. Bot. 25, Jena 1932.

Bertsch, K.: Die diluviale Flora der Schwäbischen Alb. - Ber. Dtsche Bot. Ges. 48, 1930.

BERTSCH, F.: Eine neue Interglazialflora aus Süddeutschland. - (Sonderdruck ohne Angabe der Zeitschrift), 1932. - - Eine Faulschlammbildung unter dem Travertinblock von CannstattMünster. - Jahresh. Ver. f. vaterländ. Naturk. i. Württ. 1950.

v. D. Brelie, G.: Die pollenstratigraphische Gliederung des Pleistozäns in Nordwestdeutschland. 2. Die Pollenstratigraphie im jüngeren Pleistozän. - Eiszeitalter u. Gegenw. 6, Óhringen 1950. - - Zur pollenstratigraphischen Gliederung des Pliozäns in der Niederrheinischen Bucht. - Fortschr. Geol. Rheinld. u. Westf. 4, Krefeld 1959.

v. D. Brelie, G., Kilpper, K. \& Teichmüller, R.: Das Pleistozänprofil von Frimmersdorf a. d. Erft. - Fortschr. Geol. Rheinld. u. Westf. 4, Krefeld 1959.

v. D. Brelie, G. \& Rein, U.: Die Interglazialbildungen im Niederrheinischen Diluvium. - Der Niederrhein 19, Krefeld 1952. - - Pollenanalytische Untersuchungen zur Gliederung des Pleistozäns am linken Niederrhein. - Geol. en Mijnbouw 18, 195,6.

Chanda, S.: Untersuchungen zur pliozänen und pleistozänen Floren- und Vegetationsgeschichte im Leinetal und im südwestlichen Harzvorland (Untereichsfeld). - Geol. Jb. 79, Hannover 1962.

Florschütz, F. \& van Someren, M. H.: The palaeobotanical Boundary Pliocene-Pleistocene in the Netherlands. - Internat. Geol. Congress, Report of the Eighteenth Session Great Britain 1948, Part. IX, 1950.

v. D. Hammen, T.: A contribution to the Palaeobotany of the Tiglian. - Geol. en Mijnbouw 7, 1951.

KaIser, K. \& Schütrumpf, R.: Zur Gliederung mittel- und jungpleistozäner Schichten in der Niederrheinischen Bucht. - Eiszeitalter u. Gegenw. 11, Öhringen 1960.

Kolumbe, E.: Pollenanalytische Untersuchungen an Interglazialen im Raum von Karlsruhe. - Eiszeitalter u. Gegenwart 11, Óhringen 1960. - - Die interglazialen und interstadialen Ablagerungen von Steinbach bei Baden-Baden. - Oberrhein. geol. Abh. 12, Karlsruhe 1963.

Kortenbout van Der Sluijs, G. \& Z Zagwijn, W. H.: An Introduction to the Stratigraphy and Geology of the Tegelen clay-pits. - Med. Geol. Sticht., N.S. 15, 1962.

Kuss, S. E.: Ein Beitrag zur Pleistozän-Fauna von Herxheim (Pfalz). - Ber. naturf. Ges. Freiburg i. Br. 51, 1961.

Leschiк, G.: Mikrobotanisch-stratigraphische Untersuchung der jungpliozänen Braunkohle von Buchenau (Kr. Hünfeld). - Palaeontographica 92, B, Stuttgart 1952.

Lüttig, G. \& ReIN, U.: Das Cromer (Günz/Mindel)-Interglazial von Bilshausen (Untereichsfeld). (Vorläufige Mitteilung). - Geol. Jb. 70, Hannover 1954.

Plewe, E.: Geomorphologische Studien am pfälzischen Rheingrabenrand. - Bad. geogr. Abh. 19, Freiburg i. Br. 1938.

ReIn, U.: Die pollenstratigraphische Gliederung des Pleistozäns in Nordwestdeutschland. 1. Die Pollenstratigraphie im älteren Pleistozän. - Eiszeitalter u. Gegenw. 6, Ơhringen 1955.

SzafER, W.: Pleistocene Stratigraphy of Poland from the floristical point of view. - Ann. Soc. géol. Pologne 22, Krakau 1953.

v. D. Vlerk, I. M. \& Florschütz, F.: The palaeontological base of the subdivision of the Pleistocene in the Netherlands. - Verhand. kon. nederl. Akademie van Wetenschappen, afd. Natuurkunde. Eerste Reeks, Deel XX, No. 2. Amsterdam 1953.

Woldstedt, P.: Das Eiszeitalter. Bd. I u. II. - Enke-Verlag, Stuttgart 1954 u. $195,8$.

ZAGwIJN, W. H.: Zum heutigen Stand der pollenanalytischen Untersuchungen des Pleistozäns in den Niederlanden. - Geol. en Mijnbouw 18, 1956. - - Vegetation, Climate and Time-correlations in the Early Pleistocene of Europe. - Geol. en Mijnbouw, N.S. 19, 1957 . - Aspects of the Pliocene and Early Pleistocene Vegetation in the Netherlands. - Med. geol. Sticht., Serie C-III-I, no. 5, 1960. - - Pleistocene stratigraphy in the Netherlands, based on changes in vegetation and climate. - Proc. Kon. Ned. Geol. Mijnb. Gen. 1963. - - Pollen-analytical investigations in the Tiglian of the Netherlands. - Med. geol. St. N.S. 16, 1963.

Manuspr. eingeg. 29. 5. 1965.

Anschrift des Verf.: Dr. Ilse Peters, Geograph. Institut der Universität, 53 Bonn, Franziskanerstr. 2. 